\title{
Competing desorption pathways during epitaxial growth: LEEM investigation of $\mathrm{Cu} / \mathrm{W}(110)$ heteroepitaxy
}

\author{
E. Z. Luo, Q. Cai, ${ }^{*}$ W. F. Chung \\ Department of Physics, Hong Kong University of Science and Technology, Clear Water Bay, Kowloon, Hong Kong \\ B. G. Orr \\ Department of Physics, University of Michigan, Ann Arbor, Michigan 48109-1120 \\ M. S. Altman \\ Department of Physics, Hong Kong University of Science and Technology, Clear Water Bay, Kowloon, Hong Kong
}

(Received 5 February 1996; revised manuscript received 12 August 1996)

\begin{abstract}
Competing desorption during the initial stages of epitaxial growth of $\mathrm{Cu}$ on the $\mathrm{W}(110)$ surface has been studied with low-energy electron microscopy $($ LEEM). LEEM observations of a strain-induced $(1 \times 1)-(15 \times 1)$ transformation of the $\mathrm{Cu}$ overlayer which occurs at a critical coverage of $\theta_{c}=2.13 \mathrm{ML}$ have been used as a very accurate, local probe of coverage during deposition. It is found that the growth rate vanishes at high temperature, $T>950 \mathrm{~K}$, due to competing desorption. A mean-field growth model which includes desorption and parametrization of interlayer diffusion flux quantitatively accounts for the experimentally measured dependence of the growth rate upon temperature and incident flux. Our results indicate that there is little or no $\mathrm{Cu}$ interlayer diffusion during growth. The desorption energy $E=3.67 \mathrm{eV}$ and attempt frequency $\nu=2.4 \times 10^{15}$ $\mathrm{sec}^{-1}$ are determined by examining the balance of incident and desorption fluxes. A step-flow-like growth morphology of the $(15 \times 1)$ phase occurs when the supersaturation is significantly reduced by competing desorption at high temperature. Island nucleation and coalescence is prominent in the absence of competing desorption at low temperature. [S0163-1829(96)02444-7]
\end{abstract}

\section{INTRODUCTION}

Several kinetic processes comprise epitaxial growth, including adsorption, desorption, diffusion, and nucleation and growth via islanding and step flow. ${ }^{1}$ In order to obtain highquality thin films via layer-by-layer growth, the supersaturation of the surface by the incident flux must be sufficiently low that coalescence of existing islands can occur prior to significant nucleation in subsequent layers. This can be achieved, first of all, by using low incident flux. Alternatively, enhanced adatom diffusion to existing islands and steps also reduces the supersaturation at high temperatures. Enhanced desorption at high temperature has a similar effect on the supersaturation. However, unlike diffusion, the competing desorption pathway imposes a serious physical limit to growth because it effectively reduces the overall growth rate. In the present work, we have examined the initial stages of $\mathrm{Cu} / \mathrm{W}(110)$ heteroepitaxy at high temperature with the aim of quantitative determination of desorption parametersattempt frequency and desorption energy-under epitaxial growth conditions. Using low-energy electron microscopy (LEEM), important insight into the impact of desorption and diffusion upon the growth morphology is also obtained.

Previous investigations of the $\mathrm{Cu} / \mathrm{W}(110)$ system have focused on structure and electronic properties. ${ }^{2-6}$ It was found that $\mathrm{Cu}$ initially grows pseudomorphically on the W(110) surface over a wide range of temperature. The pseudomorphic $\mathrm{Cu}$ film is distorted and laterally expanded by $25 \%$ compared to the $\mathrm{Cu}(111)$ plane. At room temperature, superstructure diffraction spots corresponding to an approximate $(15 \times 1)$ periodic structure appear in the substrate [110] direc- tion at $1-\mathrm{ML}$ coverage $\left(1 \mathrm{ML}_{\mathrm{bcc}} \equiv 1 \mathrm{ML}\right)$ and increase in intensity up to $2.13 \mathrm{ML}$. The $(15 \times 1) \mathrm{Cu}$ film is understood to have a fcc(111)-like structure with NishiyamaWassermann orientation relative to the substrate. In this orientation, the densely packed [1 10$]$ rows of the fcc overlayer are parallel to the bcc substrate [001] direction. The superperiodicity is due to the presence of a misfit dislocation consisting of one additional [110] Cu row every fifteen substrate spacings. Thus, the $(15 \times 1)$ double layer which is completed at $2.13 \mathrm{ML}$ remains laterally expanded by $17 \%$ with respect to the $\mathrm{Cu}(111)$ plane. Between 2.13 and $2.47 \mathrm{ML}[2.47$ $\mathrm{ML}_{\mathrm{bcc}}$ for $\mathrm{W}(110) \approx 2 \mathrm{ML}_{\mathrm{fcc}}$ for $\left.\mathrm{Cu}(111)\right]$, the $(15 \times 1)$ structure is gradually replaced by an approximate $(1 \times 8)$ structure. This structure consists of a double $\mathrm{Cu}$ layer with nearly undistorted $\mathrm{Cu}(111)$ periodicity. The structural transformations of $\mathrm{Cu}$ on $\mathrm{W}(110)$ are therefore understood to be a sequential relief of strain in the $\mathrm{Cu}$ film. The competition of film strain energy with the potential energy gained by placing overlayer atoms in the substrate potential minima (pseudomorphy) has been considered theoretically by Bauer and van der Merwe for fcc(111)/bcc(110) interfaces. ${ }^{7}$

At temperatures approaching $1000 \mathrm{~K}$ where our experiments were carried out, the $(1 \times 1)-(15 \times 1)$ transformation and subsequent $(15 \times 1)-(1 \times 8)$ transformation also occur. However, there have been no detailed investigations of the coverage dependence of these transformations at high temperature. As described below, our experimental investigations indicate that the onset of the $(1 \times 1)-(15 \times 1)$ transformation at high temperature is delayed until about $2 \mathrm{ML}$ prior to its completion at $2.13 \mathrm{ML}$. This $(1 \times 1)-(15 \times 1)$ transformation plays a particularly important role in our work. Direct 
observation of this transformation with LEEM provides an extremely accurate, local probe of coverage which allows quantitative modeling of the epitaxial growth process.

\section{EXPERIMENTAL RESULTS}

The $\mathrm{W}$ sample was oriented to within $0.05^{\circ}$ from the (110) plane. It was cleaned by annealing to $1000 \mathrm{~K}$ in an oxygen pressure of $1 \times 10^{-7}$ torr and flashing to $2000 \mathrm{~K}$. Copper was deposited from a resistively heated alumina crucible with a base pressure during deposition of $1 \times 10^{-9}$ torr. The experiments were carried out in a LEEM. Contrast, resolution and the operational principle of LEEM have been described previously. ${ }^{8,9}$ In summary, LEEM images surfaces with elastically backscattered, low-energy electrons. The lateral resolution of the instrument used in this work was 15 $\mathrm{nm}$. The capability to image monoatomic surface steps gives LEEM atomic resolution perpendicular to the surface. ${ }^{9}$ The high reflectivity of low-energy electrons (typically $0-30 \mathrm{eV}$ ) permits imaging in real time. The images presented in this paper were reproduced from video tape.

The experimental measurements concentrated on the $(1 \times 1)-(15 \times 1)$ transformation of the $\mathrm{Cu}$ double layer. In addition to direct observations of the growth morphology, we measured the dependence of the completion time for the $(15 \times 1)$ transformation, $t_{c}$, upon the sample temperature, $T$, and the incident flux, $F$. The completion time is equivalently the time required for the coverage to reach the $(15 \times 1) \mathrm{Cu}$ double-layer completion coverage, $\theta\left(t_{c}\right)=\theta_{c}=2.13 \mathrm{ML}$. This time is related to the net flux, $F-\theta D$, where the desorption flux, $\theta D$, is the coverage times the desorption rate, $D=\nu \exp \left(-E / k_{B} T\right)$. The desorption flux is equivalent to the desorption rate in thermal desorption assuming first-order kinetics. Of course, this expression for the net flux is an oversimplification for $\mathrm{Cu} / \mathrm{W}(110)$, for which desorption is layer dependent ${ }^{3}$ and compensation effects may occur. This expression is intended only as a schematic introduction to the phenomena of competing desorption.

LEEM observations of the $(1 \times 1)-(15 \times 1)$ transformation at 790 and $980 \mathrm{~K}$ and incident flux of $0.23 \mathrm{ML}$ per minute are shown in Figs. 1(a) -1 (c) and 1(d)-1(f), respectively. The transformations at these two temperatures are typical of the distinct growth behavior in low- and high-temperature regimes. At low temperature, nucleation of the $(15 \times 1)$ phase occurred at numerous sites on terraces and at steps and the growth proceeded by islanding and coalescence. At high temperature, nucleation occurred only at steps and growth proceeded rapidly in a smooth step-flow-like mode. At 790 $\mathrm{K}$, the $(15 \times 1)$ phase initially nucleated after $4^{\prime} 16^{\prime \prime}$ of deposition and was completed at $\theta_{c}=2.13 \mathrm{ML}$ after a total of $9^{\prime} 17^{\prime \prime}$. The completion time at $790 \mathrm{~K}$ (in the absence of desorption) calibrates the incident flux, $F=\theta_{c} / t_{c}$. Using this flux calibration, the nucleation of the $(15 \times 1)$ phase at $790 \mathrm{~K}$ is determined to occur at $0.98 \mathrm{ML}$. This is in excellent agreement with earlier studies of the transformation at low temperature. ${ }^{3}$ On the contrary, the $(15 \times 1)$ phase nucleated after $12^{\prime} 05^{\prime \prime}$ of deposition at $980 \mathrm{~K}$ and the transformation was completed at $\theta_{c}$ after an additional $47^{\prime \prime}$. The time elapsed during the transformation at $980 \mathrm{~K}$ corresponds to a deposition of 0.18 ML. However, the net amount of material collected by the surface during the transformation was actually

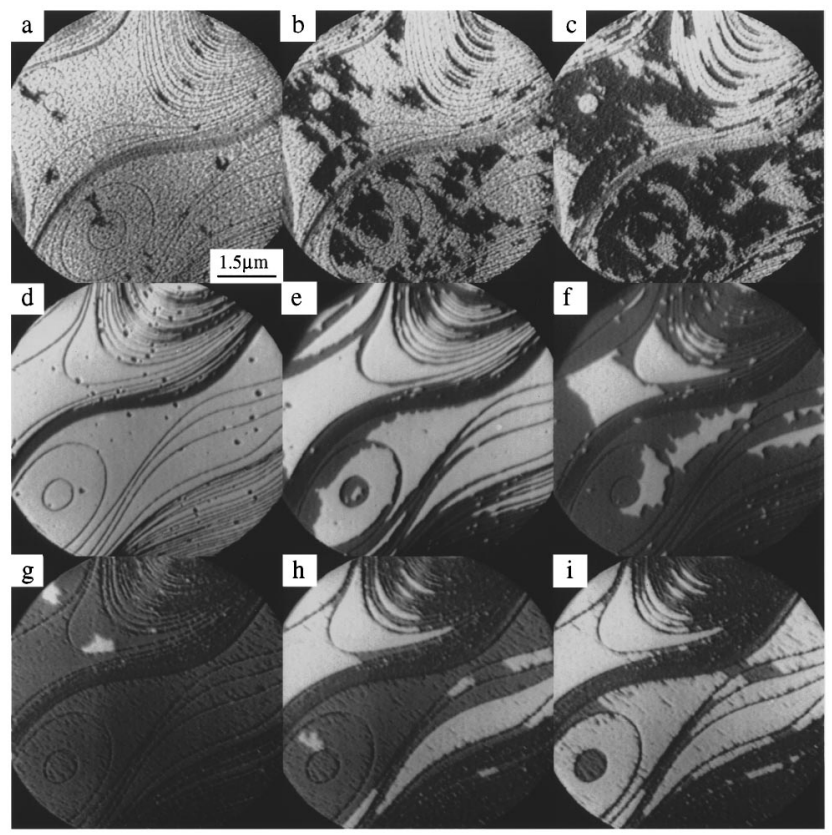

FIG. 1. LEEM images of the $\mathrm{W}(110)-\mathrm{Cu}(1 \times 1) \leftrightarrow(15 \times 1)$ transformation during (a)-(c) $\mathrm{Cu}$ deposition at $790 \mathrm{~K}$, (d)-(f) $\mathrm{Cu}$ deposition at $980 \mathrm{~K},(\mathrm{~g})-(\mathrm{i}) \mathrm{Cu}$ desorption at $980 \mathrm{~K}[(1 \times 1)=$ white; $(15 \times 1)=$ black]. Image (a) was taken after 4 min $21 \mathrm{sec}$ of deposition. The times elapsed after (a) are (b) $59 \mathrm{sec}$, (c) 2 min. Image (d) was taken after $12 \mathrm{~min} 5 \mathrm{sec}$ of deposition. The times elapsed after (d) are (e) $10 \mathrm{sec}$, (f) $25 \mathrm{sec}$. The times elapsed after (g) are (h) 43 sec, (i) $1 \mathrm{~min} 12 \mathrm{sec}$. The deposition rates in (a)-(c) and (d)-(f) were each 0.23 ML/min. Monoatomic steps are seen as dark lines.

lower than this due to concurrent desorption. This desorption was responsible for the notably longer completion time at high temperature. Additional evidence of desorption was the occurrence of the reverse $(15 \times 1)-(1 \times 1)$ transformation in the absence of incident flux. That is, the $(15 \times 1) \mathrm{Cu}$ double layer returns to the pseudomorphic structure when the coverage is reduced below $\theta_{c}$ through desorption. This $(15 \times 1)-$ $(1 \times 1)$ transformation is shown occurring at $980 \mathrm{~K}$ in Figs. $1(\mathrm{~g})-1$ (i) after the incident flux was turned off following the deposition depicted in Figs. 1(d)-1(f). On the other hand, the $(15 \times 1)$ phase was stable in the absence of an incident flux at $790 \mathrm{~K}$. This indicates that there was no significant desorption at this lower temperature.

The dependence of $t_{c}$ upon $T$ was experimentally determined for seven different incident fluxes. Typical data sets for two of these fluxes are shown in Fig. 2. The error bars in this figure are indicative of the duration of the $(15 \times 1)$ growth. The scatter of the data is believed to be related to the $\mathrm{Cu}$ source stability. Also indicated in this figure are the balance temperatures, $T_{b}$, at which the incident flux and desorption flux are equal at $\theta_{c} . T_{b}$ was determined by adjusting the sample temperature at fixed incident flux to the condition that the $(15 \times 1)$ and $(1 \times 1)$ structures coexisted. This determination of $T_{b}$ was made with a relative accuracy better than $1 \mathrm{~K}$. The main features of the data in Fig. 2 are the divergence of $t_{c}$ as $T_{b}$ is approached and the dependence of $T_{b}$ upon incident flux. The flux dependence of $T_{b}$ is discussed in greater detail below. A mean-field growth model which quantitatively accounts for these data is described next. 

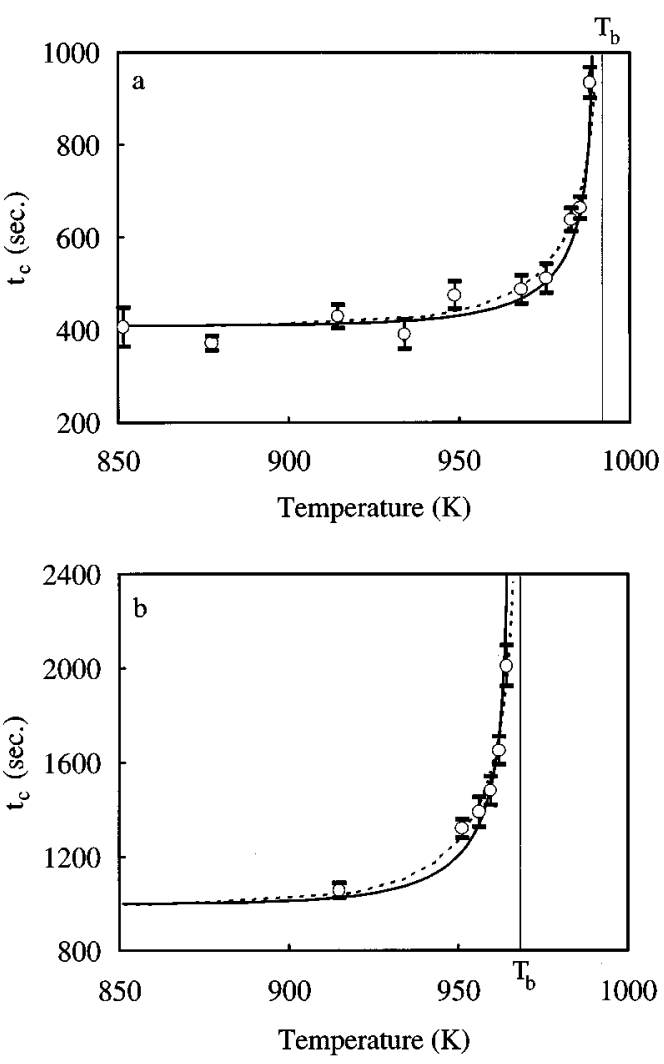

FIG. 2. Completion time of the $\mathrm{W}(110)-\mathrm{Cu}(1 \times 1)-(15 \times 1)$ transformation, $t_{c}$, versus the sample temperature for an incident flux of (a) $0.31 \mathrm{ML} / \mathrm{min}$, (b) $0.13 \mathrm{ML} / \mathrm{min}$. The vertical solid lines indicate the experimentally determined balance temperatures, $T_{b}$. The best fit of Eq. (3) (-) yields (a) $\nu_{2}=2.7 \times 10^{15} \mathrm{sec}^{-1}$, (b) $\nu_{2}=3.1 \times 10^{15} \mathrm{sec}^{-1}$. The best fit of the generalized model [Eq. (4)] with $\alpha=0$ (---) yields (a) $\nu_{2}=2.3 \times 10^{15} \mathrm{sec}^{-1}$, (b) $\nu_{2}=2.6 \times 10^{15}$ $\mathrm{sec}^{-1}$. The desorption energy is taken to be $E_{2}=3.67 \mathrm{eV}$ (see text and Fig. 5 for explanation).

\section{GROWTH MODEL AND COMPETING DESORPTION}

In order to quantitatively understand the data presented above, we have developed a mean-field growth model. Our approach is similar to rate-equation models which have been used previously to describe growth, ${ }^{10,11}$ except that we have also included competing desorption. In our model, the rate of change of the total coverage, $\theta(t)$, is equal to the incident flux minus the desorption fluxes from the first layer $(\mathrm{Cu}$ bound to the underlying $\mathrm{W}$ substrate) and from the second layer $(\mathrm{Cu}$ bound to the first $\mathrm{Cu}$ layer):

$$
\frac{d \theta(t)}{d t}=F-D_{1}\left(1-\frac{\theta_{2}(t)}{\theta_{1}(t)}\right) \theta_{1}(t)-D_{2} \theta_{2}(t),
$$

where the total coverage is the sum of the coverage in the first layer, $\theta_{1}(t)$, and the second layer, $\theta_{2}(t)$, and the term $\left[1-\theta_{2}(t) / \theta_{1}(t)\right]$ represents the fraction of the first layer which is not blocked from desorption by atoms occupying sites in the second layer. In Eq. (1), the desorption rates from the two layers are distinguished.

$$
D_{1}=\nu_{1} e^{\left(-E_{1} / k_{B} T\right)}, \quad D_{2}=\nu_{2} e^{\left(-E_{2} / k_{B} T\right)} .
$$

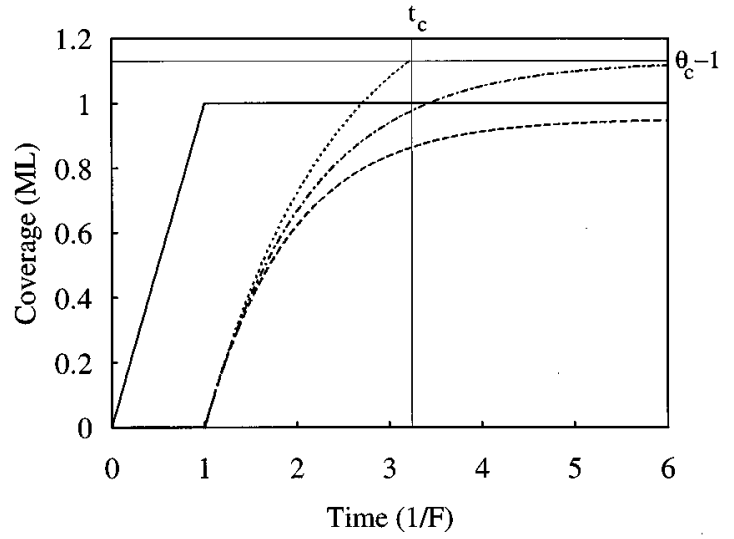

FIG. 3. Coverage take-up curves of first (-) and second $\mathrm{Cu}$ layers. The second-layer take-up curve is indicated for $T<T_{b}(\cdots)$, $T=T_{b}(-\cdots)$, and $T>T_{b}(---)$. The W(110)-Cu $(1 \times 1)$ to $(15 \times 1)$ transformation is completed at the time $t_{c}$ when the second-layer coverage reaches $\theta_{c}-1$ for $T<T_{b}$.

The distinction between first- and second-layer $\mathrm{Cu}$ desorption from the $\mathrm{W}(110)$ surface has also been made previously. ${ }^{3}$ It was found that the desorption temperature of the first $\mathrm{Cu}$ layer is about $100 \mathrm{~K}$ greater than the desorption temperature of the second $\mathrm{Cu}$ layer. Furthermore, first-layer desorption was seen to be negligibly small in the temperature range of our experiments. Therefore, first-layer desorption flux has been neglected in the following analysis.

We proceed by making the simplifying assumption that the first layer coverage increases linearly with time, $\theta_{1}(t)=F t$, and that the second layer becomes populated only after the first layer is complete at $1 \mathrm{ML}$. This is shown in the coverage take-up curves of the two layers in Fig. 3. The important physical meaning of this assumption is that all atoms which adsorb in the second layer prior to the completion of the first layer diffuse rapidly enough to the first layer to avoid desorption. (This assumption will be relaxed in a generalization of the model which is described below.) The variation of the second-layer coverage with time is found by evaluating the coverage rate equation Eq. (1). The total coverage at different times is then written as

$$
\begin{aligned}
\theta(t) & =\theta_{1}(t)=F t, \quad 0<t<\frac{1}{F} \\
\theta(t) & =1+\theta_{2}(t), \quad t>\frac{1}{F} \\
& =1+\frac{F}{D_{2}}\left(1-e^{D_{2} / F} e^{-D_{2} t}\right) .
\end{aligned}
$$

The second-layer coverage take-up curve is shown in Fig. 3 for three cases of temperature. When $T<T_{b}$, the secondlayer coverage increases until the total coverage reaches $\theta_{c}$. This defines the time $t_{c}$ at which the $(1 \times 1)-(15 \times 1)$ transformation is completed. For $T \geqslant T_{b}$, the total coverage never reaches $\theta_{c}$ and the $(15 \times 1)$ transformation is not expected to occur. This expectation is in agreement with experimental observations.

An analytical expression for $t_{c}$ is found by inverting the coverage function in Eq. (2) evaluated at $\theta_{c}$,

$$
t_{c}=\frac{1}{F}-\frac{1}{D_{2}} \ln \left[1-\left(\theta_{c}-1\right) \frac{D_{2}}{F}\right] \text {. }
$$


This expression is seen to fit the experimental data well in Fig. 2. In these fits, the desorption energy was taken to be $E_{2}=3.67 \mathrm{eV}$, while the desorption attempt frequency $\nu_{2}$ was used as fit parameter. The average value of $\nu_{2}=2.6 \times 10^{15} \pm 0.5 \times 10^{15} \mathrm{sec}^{-1}$ was obtained from fits of Eq. (3) to data sets obtained at seven different incident fluxes which ranged from 0.07 to $0.36 \mathrm{ML} / \mathrm{min}$. There was no systematic variation of $\nu_{2}$ with incident flux. We will explain below how the desorption energy was determined independently of the growth model.

In order to relax our original assumption of fast diffusion from the second layer to the first layer, we have generalized our model by parametrizing interlayer flux. In this approach, the rate equations for the coverages of the two layers are written separately.

$$
\begin{gathered}
\frac{d \theta_{1}}{d t}=F\left(1-\theta_{1}\right)+\alpha \theta_{2}\left(1-\theta_{1}\right), \\
\frac{d \theta_{2}}{d t}=F \theta_{1}-D_{2} \theta_{2}-\alpha \theta_{2}\left(1-\theta_{1}\right),
\end{gathered}
$$

As before, the desorption from the first layer has been neglected. The first term in each equation states that the direct effect of the incident flux is to increase the coverage in each layer in proportion to the fraction of available sites in that layer. The available fraction of sites in the first layer, $\left(1-\theta_{1}\right)$, decreases as the layer is filled. The available fraction of the second layer is taken to be $\theta_{1}$, rather than $\theta_{1}-\theta_{2}$. This choice automatically counts the third-layer occupancy as second-layer occupancy. Equivalently, this assumes that atoms landing in the third layer eventually either incorporate into the second layer or desorb from the third layer. This assumption is supported by experimental observations of no third-layer nucleation. Desorption from both the second and third layer is accounted for in the $D_{2} \theta_{2}$ term, which takes the desorption rates from both layers to be equal. The last term in each expression is the interlayer diffusion flux from the second layer to the first layer. The parameter $\alpha(\alpha \geqslant 0)$ specifies the magnitude of this flux. Interlayer diffusion flux from the first layer to the second layer is not considered. This is consistent with the stronger bonding of the first-layer $\mathrm{Cu}$ atoms to the $\mathrm{W}$ substrate (generally reduced mobility compared to second-layer $\mathrm{Cu}$ atoms) and the preferential attachment of atoms to steps which are encountered prior to a jump up to the next level. Further refinement of this model can be made. For example, $\alpha$ may be coverage, flux, and temperature dependent. Furthermore, compensation effects in desorption arising from interactions among particles within each layer may need to be considered. However, the results presented below suggest that additional refinements are not worthwhile.

Shown in Fig. 4 are the coverage take-up curves for the two cases of $\alpha=0$ and $\alpha=100$. These curves were determined by numerical integration of the rate Eq. (4). The case of $\alpha=0$ corresponds to no net interlayer diffusion flux. The case of $\alpha=100$ corresponds to a large net interlayer diffusion flux from the second to the first $\mathrm{Cu}$ layer. By comparison of the respective take-up curves in Figs. 3 and 4, this case is seen to be nearly equivalent to the simpler model which we described above. Although there are clear differences in the

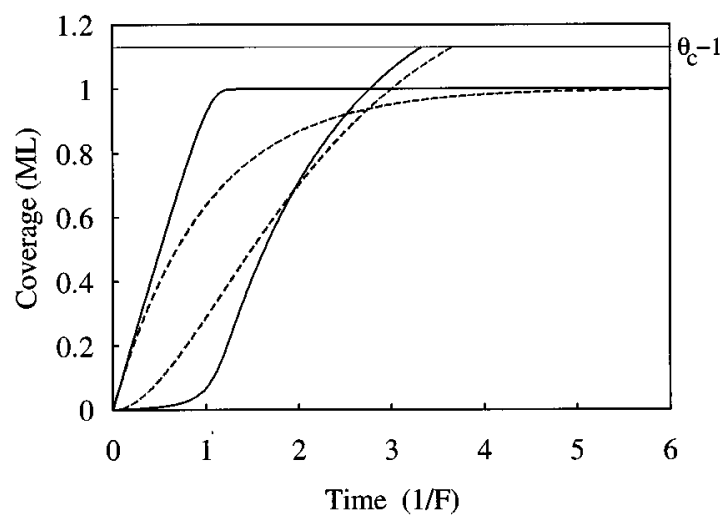

FIG. 4. Coverage take-up curves of first and second $\mathrm{Cu}$ layers for the generalized model [Eq. (4)] with the interlayer diffusion flux parametrized by $\alpha=100$ (-) and $\alpha=0$ (---).

coverage take-up curves for $\alpha=0,100$, the arrival of the total coverage at $\theta_{c}$ does not differ significantly for these two cases (Fig. 4). The distinction between our simpler model and the model parametrizing interlayer diffusion flux with $\alpha=0$ is also seen to be small in the fits to the experimental data in Fig. 2. Nevertheless, there is some indication in this figure [especially Fig. 2(b)] that the model with no interlayer diffusion flux $(\alpha=0)$ may describe the divergence of $t_{c}$ slightly better. This preference is also seen in the data obtained for other incident fluxes which are not reproduced in this paper. Inhibition of diffusion from the second to the first layer could arise from a step edge barrier to diffusion. The step edge barrier for $\mathrm{Cu}(111)$ has been determined by molecular-dynamics/Monte Carlo corrected effectivemedium theory (MD/MC-CEM) to be $176 \mathrm{meV} .{ }^{12}$ The barrier will certainly differ from $\mathrm{Cu}(111)$ and will be layer dependent on $\mathrm{Cu} / \mathrm{W}(110)$. However, our data is not of sufficient quality to quantitatively determine the step edge barrier with confidence. The model refinements mentioned above may also help to better distinguish the nature of interlayer diffusion.

The desorption energy was found by examining the flux balance condition at which the $(15 \times 1)$ and $(1 \times 1)$ phases coexist at $T_{b}$. In this condition, the desorption flux from the second layer with coverage $\theta_{2}=\theta_{c}-1$ is equal to the incident flux,

$$
\left(\theta_{c}-1\right) \nu_{2} e^{-E_{2} / k_{B} T_{b}}=F .
$$

Thus, this is essentially a conventional isosteric method ${ }^{13}$ which is independent of the growth models considered in this paper. From the flux balance condition, we find the relationship between the experimentally measured quantities $T_{b}$ and $F$ :

$$
\ln F=-\frac{E_{2}}{k T_{b}}+\ln \left[\nu_{2}\left(\theta_{c}-1\right)\right] .
$$

The desorption parameters are then determined to be $E_{2}=3.67 \pm 0.05 \mathrm{eV}$ and $\nu_{2}=2.4 \times 10^{15} \pm 1.3 \times 10^{15} \mathrm{sec}^{-1}$ by fitting this expression to the experimental data (Fig. 5). Uncertainty in the frequency arises predominantly from errors in the assignment of the desorption energy. The desorption parameters determined at the flux balance condition are pre- 


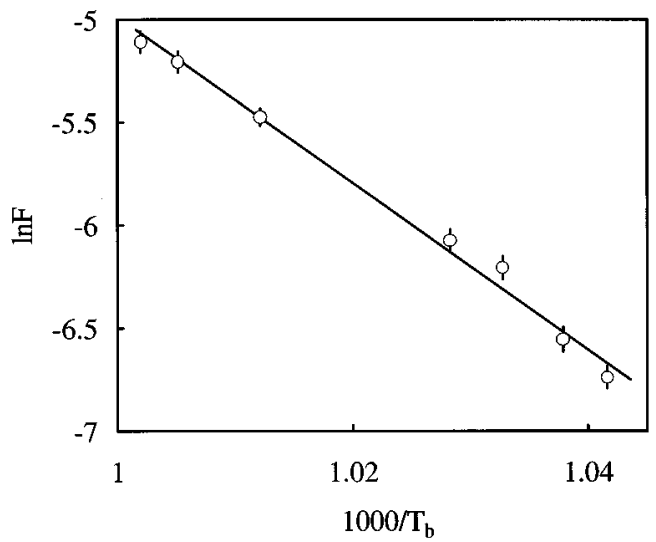

FIG. 5. Experimentally determined $T_{b}$ are plotted against the incident flux. The best fit of Eq. (5) (-) yields desorption parameters $\nu_{2}=2.4 \times 10^{15} \mathrm{sec}^{-1}$ and $E_{2}=3.67 \mathrm{eV}$.

cisely valid only for the coverage of $2.13 \mathrm{ML}$. These parameters may differ at lower coverage due to compensation effects. Nevertheless, the attempt frequency determined at flux balance is within experimental uncertainty of the value determined from the growth models above. Furthermore, unsatisfactory results were obtained when desorption energies other than $E_{2}=3.67 \mathrm{eV}$ obtained at flux balance were used in fits to the data in Fig. 2. Our result for the desorption energy can be compared to the value of $E=2.50 \mathrm{eV}$ for desorption of $\mathrm{Cu}$ from the $\mathrm{Cu}(111)$ surface determined by MD/MC-CEM. ${ }^{12}$ The large desorption energy of $\mathrm{Cu}$ from the second layer on the $\mathrm{W}(110)$ surface is consistent with the strong $\mathrm{Cu} / \mathrm{W}$ bonding which is responsible for stabilizing the $\mathrm{Cu}$ overlayer in the pseudomorphic structure. The desorption energy from the first $\mathrm{Cu}$ layer is even larger.

The desorption attempt frequency depends somewhat upon the growth model used. In particular, parametrizing interlayer diffusion flux with $\alpha=0$ yields attempt frequencies which are approximately $15 \%$ smaller than those determined with our simpler growth model (see Fig. 2). The lower values are in better agreement with the attempt frequency determined by the flux balance condition (see Fig. 5). Nevertheless, they are still about two orders of magnitude larger than desorption frequency factors which are often assumed. ${ }^{14}$ This may be due, first of all, to the sensitivity of the frequency factor to the desorption energy. We cannot rule out errors in our determination of the desorption energy. Alternatively, our result can be accounted for by transition state theory (TST) ${ }^{14}$ In TST, the prefactor appearing in the Arrhenius expression can be written in the form, $\nu=\left(k_{B} T / h\right) e^{(\Delta S / R T)}$, where $\Delta S=S_{\mathrm{tr}}-S_{i}$ is the entropy difference between the transition state and the initial state, $h$ is Planck's constant, $k_{B}$ is Boltzmann's constant, and $R$ is the universal gas constant. Setting the entropy difference to zero yields a typical prefactor of $2 \times 10^{13}$ at $1000 \mathrm{~K}$. Therefore, our enhanced value of the prefactor may be ascribed to the reduced entropy of the immobile, ordered adsorbate. Similarly enhanced desorption frequencies have been determined for several chemisorption systems, for example, $\mathrm{H} / \mathrm{Mo}(211)$ (Ref. 13) and NO/Pt(111). ${ }^{15}$ Furthermore, the $(15 \times 1)-(1 \times 1)$ restructuring of the $\mathrm{Cu}$ double-layer upon desorption may contribute to the enhanced desorption frequency. Substrate restructuring during desorption, which is neglected in TST, has been shown to cause changes in the frequency by several orders of magnitude. $^{16,17}$

\section{CONCLUSION}

We have investigated the relationship between growth morphology and supersaturation in the formation of ultrathin $\mathrm{Cu}$ films on the $\mathrm{W}(110)$ surface. These films undergo a transformation from a $(1 \times 1)$ pseudomorphic structure to a $(15 \times 1)$-periodic structure when the coverage reaches a critical value of $2.13 \mathrm{ML}$. The highest-quality $\mathrm{Cu}$ films are formed under conditions when there is significant competing desorption at high temperature. These films are characterized by a smooth step-flow-like growth of the $(15 \times 1)$ phase. In contrast, $\mathrm{Cu}$ films have many observable defects as a result of the island nucleation and coalescence growth mode which occurs in the absence of competing desorption at low temperature. Evidence of desorption at high temperature is a significant reduction of the growth rate, and the transformation of the $(15 \times 1) \mathrm{Cu}$ double layer back to the lower coverage $(1 \times 1)$ pseudomorphic double layer in the absence of an incident $\mathrm{Cu}$ flux. Mean-field growth models have been developed which predict the temperature and flux dependence of the growth rate and balance condition between incident and desorption flux. Our results indicate that there is little or no diffusion of atoms between the first and second $\mathrm{Cu}$ layers during growth, which is consistent with a step edge diffusion barrier. By exploring the balance between incident and desorption flux at the critical coverage, we have determined the desorption energy, $E_{2}=3.67 \mathrm{eV}$, and frequency, $\nu_{2}=2.4 \times 10^{15} \mathrm{sec}^{-1}$ of $\mathrm{Cu}$ atoms from the second $\mathrm{Cu}$ layer. The somewhat high attempt frequency has been considered with regard to transition state theoretical predictions and experimental results for other chemisorption systems.

\section{ACKNOWLEDGMENTS}

The authors are grateful to Greg Lopinski for stimulating discussions. Financial support was kindly provided by the Hong Kong Research Grants Council under research Grant No. HKUST642/94P.
*Permanent address: Department of Physics, Fudan University, Shanghai, China.

${ }^{1}$ W. K. Burton, N. Cabrera, and F. C. Frank, Philos. Trans. R. Soc. London, Ser. A 243, 299 (1951).

${ }^{2}$ N. Taylor, Surf. Sci. 4, 161 (1966).

${ }^{3}$ E. Bauer, H. Poppa, G. Todd, and F. Bonczek, J. Appl. Phys. 45, 5164 (1974).

${ }^{4}$ E. Bauer, Appl. Surf. Sci. 11/12, 479 (1982).
${ }^{5}$ G. Lilienkamp, C. Koziol, and E. Bauer, Surf. Sci. 226, 358 (1990).

${ }^{6}$ Y. W. Mo and F. J. Himpsel, Phys. Rev. B 50, 7868 (1994).

${ }^{7}$ E. Bauer and Jan H. van der Merwe, Phys. Rev. B 33, 3657 (1986).

${ }^{8}$ E. Bauer, Rep. Prog. Phys. 57, 895 (1994).

${ }^{9}$ M. S. Altman and W. F. Chung, Proceedings of the 13th International Congress on Electron Microscopy, Paris, 1994 (Les 
Editions des Physique, Les Ulis, 1994), pp. 1025-1026.

${ }^{10}$ P. I. Cohen, G. S. Petrich, P. R. Pukite, and G. J. Whalley, Surf. Sci. 216, 222 (1989).

${ }^{11}$ H. A. van der Vegt, H. M. van Pinxteren, M. Lohmeier, and E. Vlieg, Phys. Rev. Lett. 68, 3335 (1992).

${ }^{12}$ Y. Li and A. E. DePristo, Surf. Sci. 351, 189 (1996).

${ }^{13}$ G. Lopinski, J. A. Prybyla, and P. J. Estrup, Surf. Sci. 315, 269 (1994).
${ }^{14}$ S. J. Lombardo and A. T. Bell, Surf. Sci. Rep. 13, 1 (1991).

${ }^{15}$ R. J. Gorte, L. D. Schmidt, and J. L. Gland, Surf. Sci. 109, 367 (1981).

${ }^{16}$ V. P. Zhdanov, Surf. Sci. 209, 523 (1989).

${ }^{17}$ A. Horlacher-Smith, R. A. Barker, and P. J. Estrup, Surf. Sci. 136, 327 (1984). 